\title{
Éditorial
}

\section{Le poids de notre nation}

\author{
Hans Krueger, Ph. D. (1,2)
}

Diffuser cet article sur Twitter

En 2016, les Canadiens ont dépensé environ 228 milliards de dollars en soins de santé, soit $11,1 \%$ de notre économie totale, ou 6299 dollars par habitant. Presque 40 \% des dépenses publiques sont consacrées au

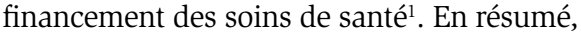
cela fait beaucoup d'argent! Ce numéro de Promotion de la santé et prévention des maladies chroniques au Canada met l'accent sur trois maladies contribuant à ce fardeau économique des soins de santé au Canada.

Selon Pelletier et ses collaborateurs ${ }^{2}$, environ 700000 Canadiens déclarent souffrir de symptômes associés au trouble d'anxiété généralisée (TAG) et, dans $30 \%$ des cas, les besoins de ces personnes en matière des soins de santé ne sont pas comblés. Amankwah et ses collègues ${ }^{3}$ estiment que 99000 Canadiens étaient atteints de sclérose en plaques en 2011 et qu'ils seront 134000 en 2031. En 2011, le montant des coûts imputables à la sclérose en plaques assumés par le système de santé et par les patients a été estimé à 1,48 milliards de dollars.

Bilandzic et Rosella ${ }^{4}$ ont calculé que 2156000 nouveaux cas de diabète seront diagnostiqués au Canada entre 2011-2012 et 2021-2022 et que les coûts de soins de santé associés atteindront 15,36 milliards de dollars, soit 7124 dollars par personne diabétique. Ils ont également estimé que 283000 cas de diabète et des coûts associés de 2,03 milliards de dollars pourraient être évités si le poids moyen des Canadiens diminuait de $5 \%$.

L'importance accordée par Bilandzic et Rosella à la relation entre diabète et surpoids est pertinente : $62 \%$ des cas de diabète de type 2 au Canada seraient attribuables à un excès de poids ${ }^{5}$. Notons que
$18 \%$ des cas seraient liés à la sédentarité et $8 \%$ au tabagisme. Or, d'après les données de l'Enquête sur la santé dans les collectivités canadiennes (ESCC), la prévalence du tabagisme chez les Canadiens de 20 à 64 ans a baissé, passant de 24,8 \% à 16,2\% entre 2001 et 2014, la prévalence de la sédentarité étant passée quant à elle de $55 \%$ à $47 \%$. Le plus grand défi demeure donc l'excès de poids, d'autant plus que, pendant la même période, la prévalence de l'obésité a augmenté, passant de 15,3\% à 20,6 \% (4 557000 Canadiens au total). Plus important encore, la prévalence liée aux niveaux d'obésité les plus élevés a plus que doublé, et on sait que le fardeau sur la santé et le fardeau économique associés à l'obésité augmentent considérablement lorsque le poids augmente.

Les tendances divergentes entre la prévalence du tabagisme et celle du surpoids au Canada ont fait que le fardeau économique imputable au surpoids est maintenant de $25 \%$ plus élevé que le fardeau économique imputable au tabagisme ${ }^{5}$. Ce croisement de tendances s'est produit en 2009 , et l'écart se creuse depuis entre les deux.

La diminution de la prévalence du tabagisme a permis de réduire de $34 \%$ le fardeau économique du Canada attribuable à ce problème entre 2000 et 2015, alors que le fardeau économique associé au surpoids a quant à lui augmenté de $24 \%$ pendant la même période. Dans ce contexte, comment pourrions-nous réussir à atteindre la baisse de poids, pourtant modeste, de $5 \%$ suggérée par Bilandzic et Rosella? Pouvonsnous appliquer au problème du surpoids les leçons tirées des réussites dans la lutte contre le tabagisme? Au cours des 60 dernières années, les progrès réalisés dans la prévention du tabagisme sont passés par une démarche globale à long terme consistant notamment à hausser les prix (habituellement par la taxation), à réglementer la publicité des produits du tabac, à diffuser des contre-publicités, à améliorer les stratégies cliniques de renoncement au tabac et à mettre en place des mesures législatives à l'égard de la qualité de l'air (interdictions de fumer) ${ }^{6}$.

Si les progrès accomplis dans la prévention du tabagisme ont demandé d'importants efforts, et quoiqu'il reste encore beaucoup de travail à faire, la lutte contre le surpoids s'annonce encore plus complexe. Si, pour simplifier à l'extrême, le surpoids découle simplement d'un déséquilibre entre les apports et les dépenses énergétiques, les facteurs influençant les problèmes de poids forment un ensemble extrêmement complexe $^{7}$. Certains organismes, tels que l'Organisation mondiale de la santé ${ }^{8}$ et les Centers for Disease Control and Prevention des États-Unis ${ }^{9}$, ont commencé à proposer une série de stratégies pour lutter contre le surpoids, qui tiennent notamment compte de la nécessité de mesurer et d'évaluer les efforts de prévention de l'obésité. Nous savons qu'une prévention efficace du surpoids à l'échelle de la population nécessitera une approche globale, multidimensionnelle, touchant de nombreuses sphères et courant sur au moins une génération, et que l'on devra constamment renforcer les modes de vie positifs grâce à un environnement favorable ${ }^{6}$.

La santé de notre nation comme l'économie de notre pays ont besoin qu'une stratégie globale à long terme de ce type soit mise en œuvre dès maintenant. Nous ne pouvons pas nous permettre d'attendre davantage. 


\section{Références}

1. Institut canadien d'information sur la santé (ICIS). Dépenses [Internet]. Ottawa (Ont.) : ICIS; 2016. En ligne à : https://www.cihi.ca/fr/depenses-et -main-doeuvre-de-la-sante/depenses

2. Pelletier L, O’Donnell S, McRae L, Grenier J. Le fardeau du trouble d'anxiété généralisée au Canada. Promotion de la santé et prévention des maladies chroniques au Canada. 2017;37(2): 60-69.

3. Amankwah $\mathrm{N}$, Marrie $\mathrm{R}$, Bancej $\mathrm{C}$ et collab. La sclérose en plaques au Canada, 2011-2031 : résultats d'une étude de modélisation par microsimulation des répercussions épidémiologiques et économiques. Promotion de la santé et prévention des maladies chroniques au Canada. 2017;37(2): 41-53.

4. Bilandzic A, Rosella L. Les coûts du diabète sur 10 ans au Canada : intégration des coûts en soins de santé imputables au diabète à un modèle de prédiction de son incidence. Promotion de la santé et prévention des maladies chroniques au Canada. 2017;37(2): 54-59.

5. Krueger H, Krueger J, Koot J. Variation across Canada in the economic burden attributable to excess weight, tobacco smoking and physical inactivity. Can J Public Health. 2015;106(4):e171-e177.

6. Krueger H, Williams, D, Kaminsky B, McLean D. The health impact of smoking and obesity and what to do about it. Toronto (Ont.) : University of Toronto Press; 2007.

7. Kumanyika S, Jeffery R, Morabia A, Ritenbaugh C, Antipatis V. Obesity prevention: the case for action. Int $\mathrm{J}$ Obesity. 2002;26:425-436.

8. Organisation mondiale de la Santé (OMS). Approches de la prévention de l'obésité de l'enfant dans la population [Internet]. Genève (Suisse) : OMS; 2012. En ligne à : http://apps.who.int /iris / bitstream/10665/85399/1 /9789242504781_fre.pdf?ua = $1 \&$ ua = 1
9. Centers for Disease Control and Prevention (CDC). Overweight and obesity: prevention strategies and guidelines [Internet]. Atlanta (GA) : CDC [mise à jour le 19 mai 2015; consultation le 30 décembre 2016]. En ligne à : https://www.cdc.gov/obesity /resources/strategies-guidelines.html 